\title{
Theoretical Calculations of Structural, Mechanical, Electronic And Optical Properties of Sn2S3 Under Pressure
}

Baishu Chen ( $\sim$ bschen@126.com)

Heilongjiang Bayi Agricultural University https://orcid.org/0000-0002-1009-4739

Wenxia Zhu

Heilongjiang Bayi Agricultural University

Chunxiang Wang

Heilongjiang Bayi Agricultural University

Chang Wang

Heilongjiang Bayi Agricultural University

Yuanzuo Li

Northeast Forestry University

Weilong Liu

Harbin Institute of Technology

\section{Research Article}

Keywords: Mechanical properties, First-principles, Sn2S3, optical properties

Posted Date: August 19th, 2021

DOI: https://doi.org/10.21203/rs.3.rs-789940/v1

License: (1) This work is licensed under a Creative Commons Attribution 4.0 International License.

Read Full License 


\title{
Theoretical calculations of structural, mechanical, electronic and optical properties of $\mathrm{Sn}_{2} \mathrm{~S}_{3}$ under pressure
}

\author{
Baishu Chen ${ }^{\mathrm{a}^{*}}$, Wenxia Zhu ${ }^{\mathrm{a}}$, Chunxiang Wang ${ }^{\mathrm{a}}$, Chang Wanga ${ }^{\mathrm{a}}$, \\ Yuanzuo Lib ${ }^{\mathrm{b}}$, Weilong Liu ${ }^{\mathrm{c}}$ \\ ${ }^{a}$ College of Science, Heilongjiang Bayi Agricultural University, \\ Daqing 163319, China \\ ${ }^{\mathrm{b} C o l l e g e}$ of Science, Northeast Forestry University, Harbin 150040, China \\ ${ }^{\mathrm{c} C o l l e g e}$ of Physics, Harbin Institute of Technology, Harbin 150001, China
}

\begin{abstract}
The pressure effect on the structural, mechanical, electronic and optical properties of $\mathrm{Sn}_{2} \mathrm{~S}_{3}$ in the pressure range of 0-35 GPa have been evaluated by means of the first-principles calculations based on the density-functional theory. The structural parameters of $\mathrm{Sn}_{2} \mathrm{~S}_{3}$ at $0 \mathrm{GPa}$ such as lattice constants and cell volumes are consistent with the previous theoretical and experiment reports. The mechanical properties about the elastic constants $\left(\mathrm{C}_{\mathrm{ij}}\right)$ and polycrystalline elastic modulus $(\mathrm{B}, \mathrm{G}$ and E) under pressure are calculated for the first time. Furthermore, the results suggest that the $\mathrm{Sn}_{2} \mathrm{~S}_{3}$ is predicted to be mechanically stable in the range of pressure from 0 to $35 \mathrm{GPa}$ in the light of the mechanical stability conditions. The $\mathrm{Sn}_{2} \mathrm{~S}_{3}$ is found to be ductile from the value of $\mathrm{B} / \mathrm{G}$. With the increasing of pressure, the ductility of $\mathrm{Sn}_{2} \mathrm{~S}_{3}$ enhances monotonously. The pressure effect on the energy band gap and density of states of $\mathrm{Sn}_{2} \mathrm{~S}_{3}$ is also discussed, which indicates that the pressure makes the band gap of $\mathrm{Sn}_{2} \mathrm{~S}_{3}$ decreased. The optical properties of $\mathrm{Sn}_{2} \mathrm{~S}_{3}$ are calculated in the range 0-35 eV, and the results show that the $\mathrm{Sn}_{2} \mathrm{~S}_{3}$ under pressure has stronger visible light absorption in comparison with $0 \mathrm{GPa}$.
\end{abstract}

Keywords: Mechanical properties; First-principles; $\mathrm{Sn}_{2} \mathrm{~S}_{3}$; optical properties

* Corresponding author.

E-mail address: bschen@126.com (Baishu Chen). 


\section{Introduction}

The tin sulfides which consist of abundant and environmentally friendly elements have recently attracted great interest in view of the promising applications in photovoltaic absorber[1,2], photodetector[3,4], the electrode materials of lithium-ion batteries[5,6], photocatalysis[7,8], etc. The tin sulfides exist in the three stable compounds such as $\mathrm{SnS}, \mathrm{Sn}_{2} \mathrm{~S}_{3}$ and $\mathrm{SnS}_{2}$. Among them, the $\mathrm{Sn}_{2} \mathrm{~S}_{3}$ is also considered as an excellent photovoltaic material because of the reasonable density of carriers, high carrier mobility and proper band gap[9-11]. However, the relevant properties of the $\mathrm{Sn}_{2} \mathrm{~S}_{3}$, which are not well understood, is relatively poorly studied as compared to that of $\mathrm{SnS}$ and $\mathrm{SnS}_{2}$.

Earlier work shows that the $\mathrm{Sn}_{2} \mathrm{~S}_{3}$ exhibits an orthorhombic phase (Pnma, space group 62) with lattice constants $a=8.869 \AA, b=3.784 \AA, c=14.021 \AA[12]$. J.S. David reported the electronic and optical properties of $\mathrm{Sn}_{2} \mathrm{~S}_{3}$ using the first-principles calculations within the general potential linearized augmented planewave method[13]. They found that the $\mathrm{Sn}_{2} \mathrm{~S}_{3}$ is a direct forbidden semiconductor with a band gap of $0.97 \mathrm{eV}$. It has been demonstrated from experiment that the $\operatorname{Sn}_{2} \mathrm{~S}_{3}$ has a bandgap of 0.9-2.2 eV [14-16], where the difference of the bandgap in these papers is closely related with the difference in the growth direction of the single crystals and thin films synthesized in the experiment. The optical properties and room temperature conductivity of $\mathrm{Sn}_{2} \mathrm{~S}_{3}$ have been investigated by the ultraviolet-visible absorption spectroscopy and four-probe method, respectively[16]. S. Wataru et al. reported the synthesis and thermoelectric properties of $\mathrm{Sn}_{2} \mathrm{~S}_{3}$ polycrystalline samples [17]. Other studies of $\mathrm{Sn}_{2} \mathrm{~S}_{3}$ have been performed using the Raman and infrared spectra[18], X-ray photoemission spectroscopy[19], Mossbauer spectroscopy[20].

The above discussion reveals that there is no experimental or theoretical work for the elastic constants and mechanical properties of $\mathrm{Sn}_{2} \mathrm{~S}_{3}$. The elastic constants can provide the important information regarding the mechanical properties of materials, which are very fundamental for the practical applications and the development of the material. It is obvious to all that the properties of materials such as the crystallographic, mechanical and electronic properties can be modulated by high pressure[21,22]. It is highly desirable to investigate the effects of pressure on the structural, mechanical, electronic and optical properties of $\mathrm{Sn}_{2} \mathrm{~S}_{3}$. Therefore, in this work, we have systematically investigated the structural, mechanical, electronic and optical properties of $\mathrm{Sn}_{2} \mathrm{~S}_{3}$ in 
the range of pressure from 0 to $35 \mathrm{GPa}$ using the plane-wave pseudo-potentials method within the framework of the density functional theory.

\section{2 . Computational method}

In this work, the first-principles study were carried out by using the Cambridge Serial Total Energy Package (CASTEP) software[23] on the basis of the plane-wave pseudo-potentials method. The ultrasoft pseudo-potentials were chosen to deal with the exchange-correlation interaction[24]. The electronic states $\mathrm{Sn} 5 \mathrm{~s}^{2} 5 \mathrm{p}^{6}$ and $\mathrm{S} 3 \mathrm{~s}^{2} 3 \mathrm{p}^{4}$ were select as valence states. For the exchange-correlation potential, the Perdew-Burke-Ernzerhof (PBE)[25] functional and Perdew Burke-Ernzerhof potential of solid (PBEsol) functional[26] were employed in the course of geometric optimization, respectively. The plane-wave cutoff energy of $500 \mathrm{eV}$ was employed during the entire calculations. The Broyden-Fletcher-Goldfarb-Shannon (BFGS) optimization scheme was adopted to optimize the structural parameters of the compound. The integration over the Brillouin zone was described using the k-points grids of $8 \times 16 \times 6$ introduced by Monkhorst-Pack[27]. The convergent thresholds of geometry optimization are that the total energy,

the forces on atoms and the maximum stress is $1 \times 10^{-6} \mathrm{eV} / \mathrm{atom}, 0.03 \mathrm{eV} / \AA$ and $0.02 \mathrm{GPa}$, respectively.

\section{Results and discussion}

\subsection{Structural properties}

Fig.1 shows the structural model of $\mathrm{Sn}_{2} \mathrm{~S}_{3}$, which has eight $\mathrm{Sn}$ atoms and twelve $\mathrm{S}$ atoms in the unit cell. The equilibrium lattice parameters of $\mathrm{Sn}_{2} \mathrm{~S}_{3}$ on the PBE and PBEsol calculations at 0 GPa are presented in Table 1. The available theoretical and experimental results are also listed in Table 1. It is observed that the calculated lattice constants on the PBEsol method presents $1.7 \%$ of the maximal deviation from the experimental one[12], which is less than $3.0 \%$ of the PBE method. Therefore, the PBEsol method can obtain more accurate results in comparison with the PBE method for the structural properties of $\mathrm{Sn}_{2} \mathrm{~S}_{3}$. Meantime the results of the PBEsol method are consistent with the previous theoretical results[28,29]. In addition, the PBEsol method is also used to study the related properties under pressure because that there's no more available results for comparison.

Next, the crystal structure of $\mathrm{Sn}_{2} \mathrm{~S}_{3}$ under pressure is analyzed. The relative lattice constants $\left(a / a_{0}, \mathrm{~b} / \mathrm{b}_{0}, c / c_{0}\right.$ and $\left.V / V_{0}\right)$ are shown in Fig.2 as a function of pressure concentration. As shown in 
Fig. 2, The relative lattice constants $\left(a / a_{0}, \mathrm{~b} / \mathrm{b}_{0}, c / c_{0}\right.$ and $\left.V / V_{0}\right)$ decrease with the increasement of the pressures. Obviously, as the pressure increases, the ratio a/a $\mathrm{a}_{0}$ decreases most quickly, followed by $c / c_{0}$, and $b / b_{0}$ decreases most slowly. So the $\mathrm{Sn}_{2} \mathrm{~S}_{3}$ is the most difficultly compressed along the $\mathrm{b}$ axis, while the $\mathrm{Sn}_{2} \mathrm{~S}_{3}$ is the most easily compressed along the a-axis, which maybe due to the result that the Sn-S bonds along the $\mathrm{b}$ axis is shorter than the $\mathrm{Sn}-\mathrm{S}$ bonds along the a axis. This results reveals that the $\mathrm{Sn}_{2} \mathrm{~S}_{3}$ is the anisotropy of compressibility.

\subsection{Elastic and mechanical properties}

The elastic constants are the fundamental factors that determine the resistance of the applied stress, and related to the mechanical properties of the materials for instance bulk modulus B, shear modulus G, young's modulus E. As far as we known, there is no theoretical and experiment reports for the mechanical properties of $\mathrm{Sn}_{2} \mathrm{~S}_{3}$. The elastic constants of $\mathrm{Sn}_{2} \mathrm{~S}_{3}$ are calculated, when the stress-strain method was applied to the optimized structure in the light of Hooke's law[30]. There are nine independent elastic constants for the orthorhombic $\mathrm{Sn}_{2} \mathrm{~S}_{3}$, such as $C_{11}, C_{22}, C_{33}, C_{12}, C_{13}$, $C_{23}, C_{44}, C_{55}, C_{66}$. For a stable orthorhombic structure, the nine independent elastic constants should accord with the requirements of mechanical stability as follows[31]:

$$
\begin{gathered}
\tilde{C}_{i i}>0,(i=1 \sim 6), \quad \tilde{C}_{11}+\tilde{C}_{22}+\tilde{C}_{33}+2\left(\tilde{C}_{12}+\tilde{C}_{13}+\tilde{C}_{23}\right)>0, \tilde{C}_{11}+\tilde{C}_{22}-2 \tilde{C}_{12}>0 \\
\tilde{C}_{11}+\tilde{C}_{33}-2 \tilde{C}_{13}>0, \tilde{C}_{22}+\tilde{C}_{33}-2 \tilde{C}_{23}>0
\end{gathered}
$$

Here, $\tilde{C}_{i i}=C_{i i}-P>0,(i=1 \sim 6), \quad \tilde{C}_{12}=C_{12}+P, \quad \tilde{C}_{13}=C_{13}+P, \tilde{C}_{23}=C_{23}+P$, and $\mathrm{P}$ is the applied pressure.

The influnence of the pressure on the elastic constants for the $\mathrm{Sn}_{2} \mathrm{~S}_{3}$ is ploted in Fig. 3. From Fig. 3, all the elastic constants increase as the pressure increases. By calculating the mechanical stabilities under pressure, it is seen that the calculated elastic constants of $\mathrm{Sn}_{2} \mathrm{~S}_{3}$ concide with the requirements of the above mechanical stability in the range of 0-35 GPa, suggesting that the $\mathrm{Sn}_{2} \mathrm{~S}_{3}$ is stabilized mechanically in the range of the considered pressure.

Generally, the elastic modulus is represent by the bulk modulus (B), shear modulus (G) and Young's modulus $(E)$, which can be estimated through the independent single-crystal elastic constants on the base of the Voigt-Reuss-Hill (VRH) averaging scheme[32]. For the orthorhombic crystals, the bulk modulus (B), shear modulus $(\mathrm{G})$ and Young's modulus $(E)$ can be computed using the following formulas[33]: 


$$
\begin{gathered}
B_{\mathrm{V}}=(1 / 9)\left[C_{11}+C_{22}+C_{33}+2\left(C_{12}+C_{13}+C_{23}\right)\right] \\
G_{\mathrm{V}}=(1 / 15)\left[C_{11}+C_{22}+C_{33}-\left(C_{12}+C_{13}+C_{23}\right)+3\left(C_{44}+C_{55}+C_{66}\right)\right] \\
B_{\mathrm{R}}=1 /\left[\mathrm{S}_{11}+S_{22}+S_{33}+2\left(S_{12}+S_{13}+S_{23}\right)\right] \\
G_{\mathrm{R}}=15 /\left[4\left(S_{11}+S_{22}+S_{33}-S_{12}-S_{13}-S_{23}\right)+3\left(S_{44}+S_{55}+S_{66}\right)\right] \\
B=(1 / 2)\left(B_{\mathrm{V}}+B_{\mathrm{R}}\right) \\
G=(1 / 2)\left(G_{\mathrm{V}}+G_{\mathrm{R}}\right) \\
E=\frac{9 B G}{3 B+G}
\end{gathered}
$$

Where the $S_{i j}$ is the elastic compliance constant.

The variations of the calculated bulk modulus, shear modulus and Young's modulus for the $\mathrm{Sn}_{2} \mathrm{~S}_{3}$ with pressure are shown in Fig. 4. It is well known that the bulk modulus B, shear modulus G and Young's modulus $\mathrm{E}$ is in connection with the resistance to stretching deformation, the resistance to shear deformation and the stiffness of the solid. The larger the elastic modulus is, the harder the material is[34]. The results of Fig. 4 shows that all the elastic modulus increase slowly with the increase of the applied pressure, suggesting that the resistance to stretching and shear deformation, stiffness and hardness of $\mathrm{Sn}_{2} \mathrm{~S}_{3}$ is improved under pressure.

The brittleness and ductility of the materials is differentiated by the ratio of the bulk to shear modulus B/G. According to the Pugh parameter[35], if the $\mathrm{B} / \mathrm{G}$ value is more than 1.75 , the material is considered as a ductile material. Otherwise it behaves in a brittle manner. The larger the value of the $B / G$, the higher the ductility of materials. Fig. 5 presents the $B / G$ ratios of $\mathrm{Sn}_{2} \mathrm{~S}_{3}$ as a function of pressures. It is noted from Fig. 5 that the $\mathrm{B} / \mathrm{G}$ ratios are more than 1.75 , showing that the $\mathrm{Sn}_{2} \mathrm{~S}_{3}$ exhibits the ductile behavior in nature. The $\mathrm{B} / \mathrm{G}$ ratios increases monotonically when the pressure increases, which implies that the ductility of $\mathrm{Sn}_{2} \mathrm{~S}_{3}$ is enhanced as the pressure increases.

\subsection{Electronic properties}

In Fig. 6, the band gap of $\mathrm{Sn}_{2} \mathrm{~S}_{3}$ at $0 \mathrm{GPa}$ is $0.35 \mathrm{eV}$, which is smaller than the result in experiment. It is well known that the GGA approximation underestimates the band gap of the material. However, we studied mainly the relative change of the electronic properties of $\mathrm{Sn}_{2} \mathrm{~S}_{3}$ under pressure. So the calculation results in the same calculation parameters are comparable. For the sake 
of studying the pressure effect on the band gap in detail, Fig. 6 shows the pressure dependence of the calculated band gap of $\mathrm{Sn}_{2} \mathrm{~S}_{3}$. It is found that the band gap of $\mathrm{Sn}_{2} \mathrm{~S}_{3}$ decreases with increasing the pressure, which indicates that the pressure can induce a transition from a semiconducting to metallic state in $\mathrm{Sn}_{2} \mathrm{~S}_{3}$ when the pressure increases enough.

To understand detailly the band structure under pressure, the total $(0,10,20$ and $30 \mathrm{GPa})$ and partial (0 and $30 \mathrm{GPa}$ ) density of states (DOS) of $\mathrm{Sn}_{2} \mathrm{~S}_{3}$ are presented in Fig. 7 and Fig. 8, respectively. It is obvious from Fig. 7 that the band of $\mathrm{Sn}_{2} \mathrm{~S}_{3}$ can be separated into three subband. From the partial DOS of Fig. 8, it can be seen that the lower valence bands from about -16.5 to $-12.0 \mathrm{eV}$ originate considerably from $\mathrm{S} 3 \mathrm{~s}$ states. The upper valence bands between about -10.0 and $0 \mathrm{eV}$ is mainly contributed by the $\mathrm{Sn} 5 \mathrm{~s}, \mathrm{Sn} 5 \mathrm{p}$ and $\mathrm{S} 3 p$ states, where there exists the hybridization between Sn $5 p$ and S 3p states, which suggests the covalent bonding between $\mathrm{Sn}$ and $\mathrm{S}$ atoms. The conduction bands above the Fermi energy result mainly from the contributions from Sn 5p states.

It is clear to see from Fig. 7 that the total DOS of $\mathrm{Sn}_{2} \mathrm{~S}_{3}$ is influenced considerably by the pressure. With the increasing pressure, the peak value of total DOS becomes lower while the bandwidth of total DOS gets wider. It is seen from the partial DOS in Fig. 8 that the increasement of the bandwidth are mainly derived from the results that the band bottoms of the $\mathrm{S} 3$ s states and $\mathrm{Sn}$ 5s states move towards the lower energy level, while the conduction band tops of the $\mathrm{Sn} 5 \mathrm{~s}$ states move towards the higher energy level. Moreover, the $\mathrm{Sn} 5$ s states hybridize strongly with the S 3p states in the bottom part of the conduction band. The hybridization peak gets lower and shows the lower energy level shift, which makes the band gap to reduce with increasing the pressure.

\subsection{Optical properties}

The optical properties of the material are very important for the potential use of the photovoltaic material. The optical properties of the material can be usually represented by the dielectric function $\varepsilon(\omega)=\varepsilon_{1}(\omega)+i \varepsilon_{2}(\omega)$, where $\omega$ is the frequency of the light. The imaginary part $\varepsilon_{2}(\omega)$ is derived by calculating the momentum matrix elements between occupied states and unoccupied states. The real part $\varepsilon_{1}(\omega)$ is obtained using the imaginary part $\varepsilon_{2}(\omega)$ by means of the Kramer-Kronig dispersion relation. The absorption spectrum is estimated from the real parts $\varepsilon_{1}(\omega)$ and imaginary parts $\varepsilon_{2}(\omega)$ by the relation[36]: 


$$
\alpha(\omega)=\sqrt{2} \omega\left[\sqrt{\varepsilon_{1}^{2}(\omega)+\varepsilon_{2}^{2}(\omega)}-\varepsilon_{1}(\omega)\right]^{\frac{1}{2}}
$$

The previous theoretical works have revealed that the optical absorption spectrum of the material can make a comparison of the optical properties of the material[37,38]. Fig. 9 shows the absorption spectra of $\mathrm{Sn}_{2} \mathrm{~S}_{3}$ as a function of wavelength at various pressures. The absorption spectra at zero pressure exhibits two main peaks at about $113 \mathrm{~nm}$ and $177 \mathrm{~nm}$, in which the former is more prominent than the latter. The peak at the higher wavelength becomes weaker when pressure is enhanced, and disappears at $20 \mathrm{GPa}$. The peak at the lower wavelength increases slightly with increasing the pressure. The higher edge of absorption spectra for $\mathrm{Sn}_{2} \mathrm{~S}_{3}$ under pressures moves towards the visible light range below $500 \mathrm{~nm}$. The reason is that the pressure makes the band gap of $\mathrm{Sn}_{2} \mathrm{~S}_{3}$ smaller, which results in the energy required for the transition from the valence band to the conduction band decreases, so that the peak edge move towards the higher wavelength. The absorption increases obviously at the wavelengths ranging from $500 \mathrm{~nm}$ to $850 \mathrm{~nm}$ with increase in pressure. Therefore, the $\mathrm{Sn}_{2} \mathrm{~S}_{3}$ under pressures broadens the light absorption range and meanwhile enhances the light absorption, which improves the photocatalysis of the $\mathrm{Sn}_{2} \mathrm{~S}_{3}$.

\section{Conclusions}

In summary, using first-principles calculations within the Generalized Gradient Approximation (GGA) approaches, we have systematically investigated the structural, mechanical, electronic and optical properties of $\mathrm{Sn}_{2} \mathrm{~S}_{3}$ under different pressures. The calculated lattice parameters and cell volumes of $\mathrm{Sn}_{2} \mathrm{~S}_{3}$ decrease with different rates as the pressure increases. It is found that the $\mathrm{b}$ axis of $\mathrm{Sn}_{2} \mathrm{~S}_{3}$ is the most difficultly compressed and the a-axis is the most easily compressed. Effects of pressure on the elastic constants, the bulk modulus, the shear modulus, Young's modulus and the ductile or brittle character are studied in detail for the first time. The increase in pressure gives rise to the enhancements of mechanical properties. The energy bandgap and density of states of $\mathrm{Sn}_{2} \mathrm{~S}_{3}$ under pressure indicate that the $\mathrm{Sn}_{2} \mathrm{~S}_{3}$ can exhibit the transition from a semiconducting to metallic behaviour when the pressure is high enough, which results from the strong hybridization between the Sn $5 \mathrm{~s}$ states and the S 3p states in the bottom part of the conduction band. As the pressure increases, the absorption spectra of $\mathrm{Sn}_{2} \mathrm{~S}_{3}$ exhibits an obviously red shift, and the light absorption of $\mathrm{Sn}_{2} \mathrm{~S}_{3}$ was enhanced. 
Funding This work was funded by Heilongjiang Bayi Agricultural University Support Program for San Heng San Zong (Grant No.ZRCPY201817).

Conflicts of interest/Competing interests Not applicable

Availability of data and material Not applicable

Code availability Not applicable

Authors' contributions Baishu Chen and Chunxiang Wang performed all the calculations, drafted the manuscript. Wenxia Zhu and Chang Wang prepared the figures and tables. Yuanzuo Li and Weilong Liu performed the critical revision of the manuscript. All authors participated in the interpretation of the results. All authors read and approved the final manuscript.

\section{References}

[1] P. D. Antunez, J. J. Buckly, R. L. Brutchey, Nanoscale 3 (2011) 2399-2411.

[2] G.H. Yue, W. Wang, L.S. Wang, X. Wang, P.X. Yan, Y. Chen, D.L. Peng, J. Alloy Compd. 474 (2009) 445-449.

[3] M.S. Mahdi, H.S. A1-Arab, H.S. A1-Salman, K. Ibrahim, N.M. Ahmed, A. Hmood, M. Bououdina, Mater. Lett. 273 (2020) 127910-127913.

[4] G. X. Su, V. G. Hadjiev, P. E. Loya, J. Zhang, S. D. Lei, S. Maharjan, P. Dong, P. M. Ajayan, J. Lou, H. B. Peng, Nano Lett. 15 (2015) 506-513.

[5] X.S. Lv, W. Wei, H.C. Yang, J.J. Li, B.B. Huang, Y. Dai, Chem. Eur. J. 24 (2018) 11193-11199.

[6] Y.G. Zhou, J. Mater. Chem. A 4 (2016) 10906-10913.

[7] L.A. Burton, T.J. Whittles, D. Hesp, W.M. Linhart, J.M. Skelton, B. Hou, R.F. Webster, G.O.

Dowd, C. Reece, D. Cherns, D.J. Fermin, T.D. Veal, V.R. Dhanak, A. Walsh, J. Mater. Chem. A 4 (2016) 1312-1318.

[8] S.S. Hegde, B.S. Surendra, V. Talapatadur, P. Murahari, K. Ramesh, Chem. Phys. Lett. 754 (2020) 137665-137671.

[9] R.N. Mohan, M.T.S. Nair, P.K. Nair, Appl. Surf. Sci. 504 (2020) 144162-144171.

[10] L.A. Burton, D. Colobara, R.D. Abellon, F.C. Grozema, L.M. Peter, T.J. Savenije, G. Dennler, A. Walsh, Chem. Mater. 25 (2013) 4908-4916.

[11] B. Chen, X. Xu, F. Wang, J. Liu, J. Ji, Mater. Lett. 65 (2011) 400-402. 
[12] M. Cruz, J. Morales, J.P. Espinos, J. Sanz, J. Solid State Chem. 175 (2003) 359-365.

[13] J.S. David, Appl. Phys. Lett. 109 (2016) 032102-032106.

[14] U.V. Alpen, J. Fenner, E. Gmelin, Mater. Res. Bull. 10 (1975) 175-180.

[15] H.B.H. Salah, H. Bouzouita, B. Rezig, Thin Solid Films 480-481 (2005) 439-442.

[16] M. Khadraoui, N. Benramdane, C. Mathieu, A. Bouzidi, R. Miloua, Z. Kebbab, K. Sahraoui, R. Desfeux, Solid State Commun. 150 (2010) 297-300.

[17] S. Wataru, H. Kei, N. Hiroki, Y. Miyazaki, Jpn. J. Appl. Phys. 56 (2017) 061201-061204.

[18] J.M. Skelton, L.A. Burton, A. J. Jackson, F. Oba, S. C. Parkera, A. Walsh, Phys. Chem. Chem. Phys. 19 (2017) 12452-12465.

[19] J.W. Thomas, A.B. Lee, M.S. Jonathan, W. Aron, D.V. Tim, R.D. Vin, Chem. Mater. 28 (2016) 3718-3726.

[20] G. Amthauer, J. Fenner, S. Hafner, W. B. Holzaptel, R. Keller, J. Chem. Phys. 70 (1979) 4837-4842.

[21] Y.O. Ciftci, M. Evecen, I.O. Alp, Journal of Molecular Modeling, 27 (2021) 7-12.

[22] S.A.Khandy, I. Islam, D.C. Gupta, A. Laref, Journal of Molecular Modeling, 24 (2018) $131-134$.

[23] M.D. Segall, P.J.D. Lindan, M.J. Probert, C.J. Pickard, P.J. Hasnip, S.J. Clark, M.C. Payne, J. Phys.: Condens. Matter 14 (2002) 2717-2744.

[24] D. Vanderbilt, Phys. Rev. B 41 (1990) 7892-7895.

[25] J.P. Perdew, K. Burke, M. Ernzerhof, Phys. Rev. Lett. 77 (1996) 3865-3868.

[26]J.P. Perdew, A. Ruzsinszky, G.I. Csonka, O.A. Vydrov, G.E. Scuseria, L.A. Constantin, X. Zhou, K. Burke, Phys. Rev. Lett. 100 (2008) 136406-136409 .

[27] H.J. Monkhorst, J.D. Pack, Phys. Rev. B 13 (1976) 5188-5192 .

[28] A. B. Lee, W. Aron , J. Phys. Chem. C 116 (2012) 24262-24267.

[29] K. Yu, A.B. Lee, W. Aron, O. Fumiyasu, Phys. Rev. Applied 6 (2016) 014009-014022.

[30] J.F. Nye, Physical Properties of Crystals, Oxford University Press, Oxford, 1985.

[31]F. Mouhat, F.X. Coudert, Phys. Rev. B 90 (2014) 224104-224107.

[32] R. Hill, Proc. Phys. Soc. Sect. A 65 (1952) 349-354. 
[33] R. Golesorkhtabar, P. Pavone, J. Spitaler, P. Puschnig, C. Draxl, Comput. Phys. Commun. 184 (2013) 1861-1873 .

[34] F.M. Gao, J.L. He, E.D. Wu, S.M. Liu, D.L. Yu, Y.J. Tian, Phys. Rev. Lett. 91 (2003) 015502-015505 .

[35] S.F. Pugh, Phil. Mag. 4 (1954) 5 823-843.

[36] S. Saha, T.P. Sinha, A. Mookerjee, Phys. Rev. B 62 (2000) 8828-8834.

[37] H.H. Ibrahim, A.A. Mohamed, I.A. Ibrahim, Mater.Chem. Phys. 252 (2020) 123285-123293.

[38] R.J. Sa,WY, Zha D.W. Liu, J. Phys. Chem. Solids 145 (2020) 109575-109579. 


\section{Figures:}

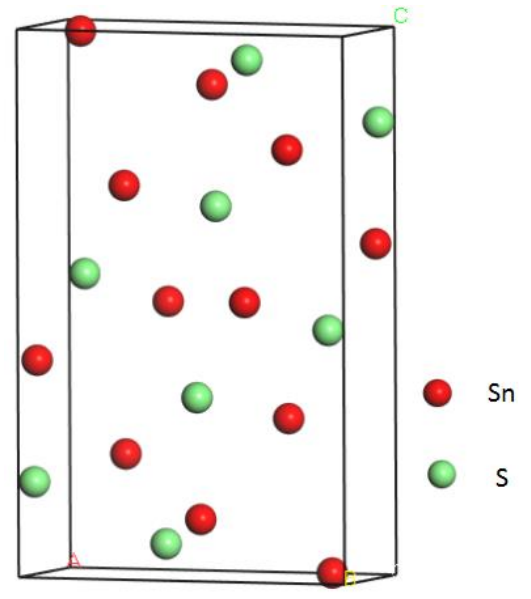

Fig. 1. The crystallographic structure of $\mathrm{Sn}_{2} \mathrm{~S}_{3}$.

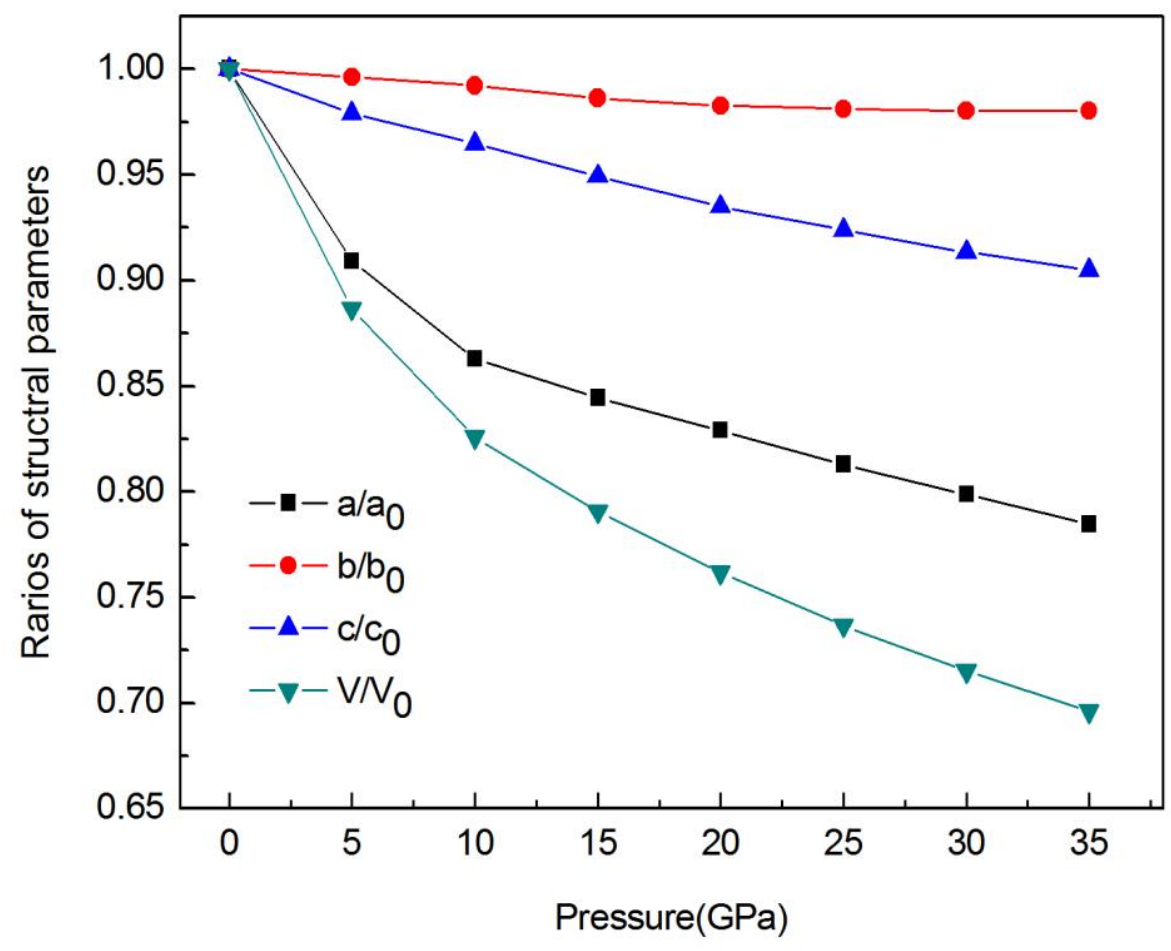


Fig. 2. The structural parameters $\mathrm{a} / \mathrm{a}_{0}, \mathrm{~b} / \mathrm{b}_{0}, \mathrm{c} / \mathrm{c}_{0}$ and $\mathrm{V} / \mathrm{V}_{0}$ as a function of the pressure for $\mathrm{Sn}_{2} \mathrm{~S}_{3}$.

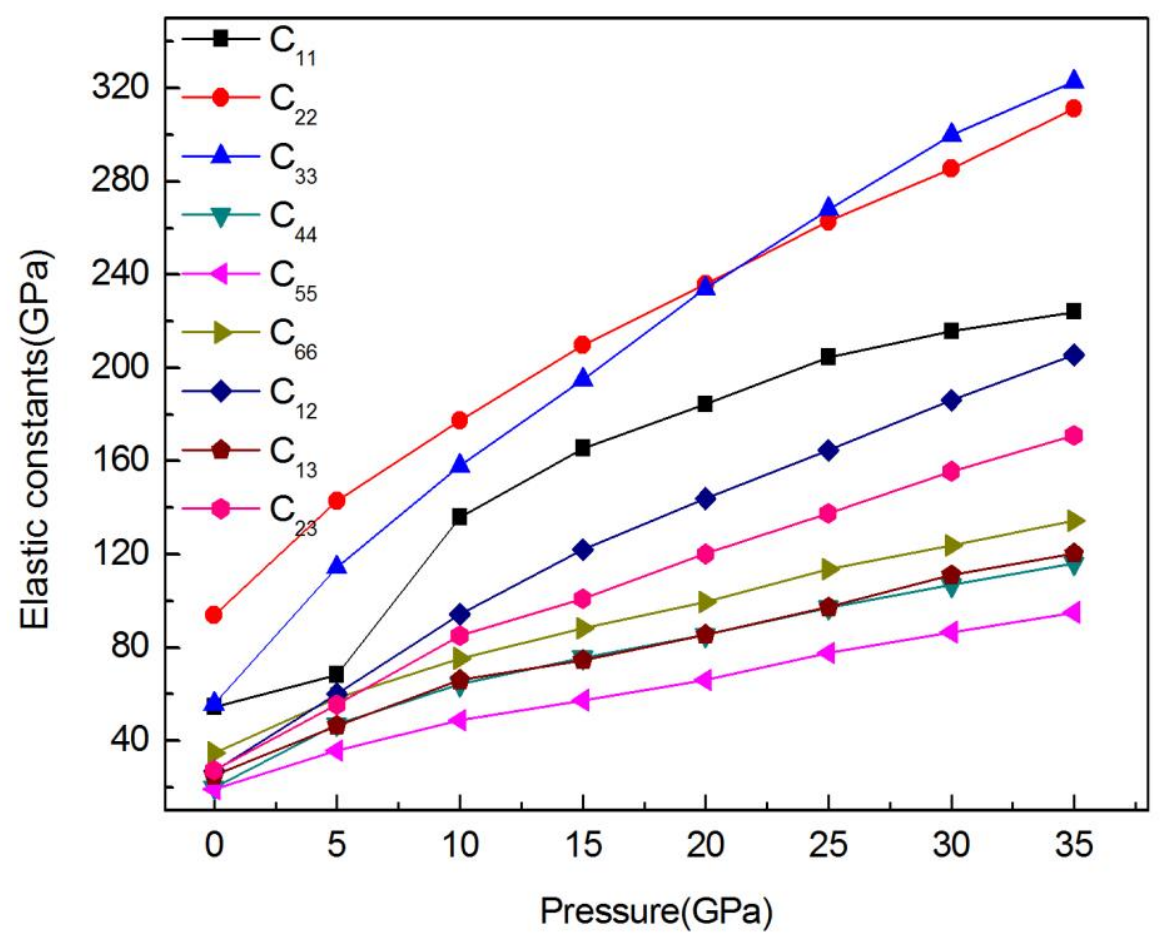

Fig. 3. The variation of the elastic constants for the $\mathrm{Sn}_{2} \mathrm{~S}_{3}$ with respect to pressure. 


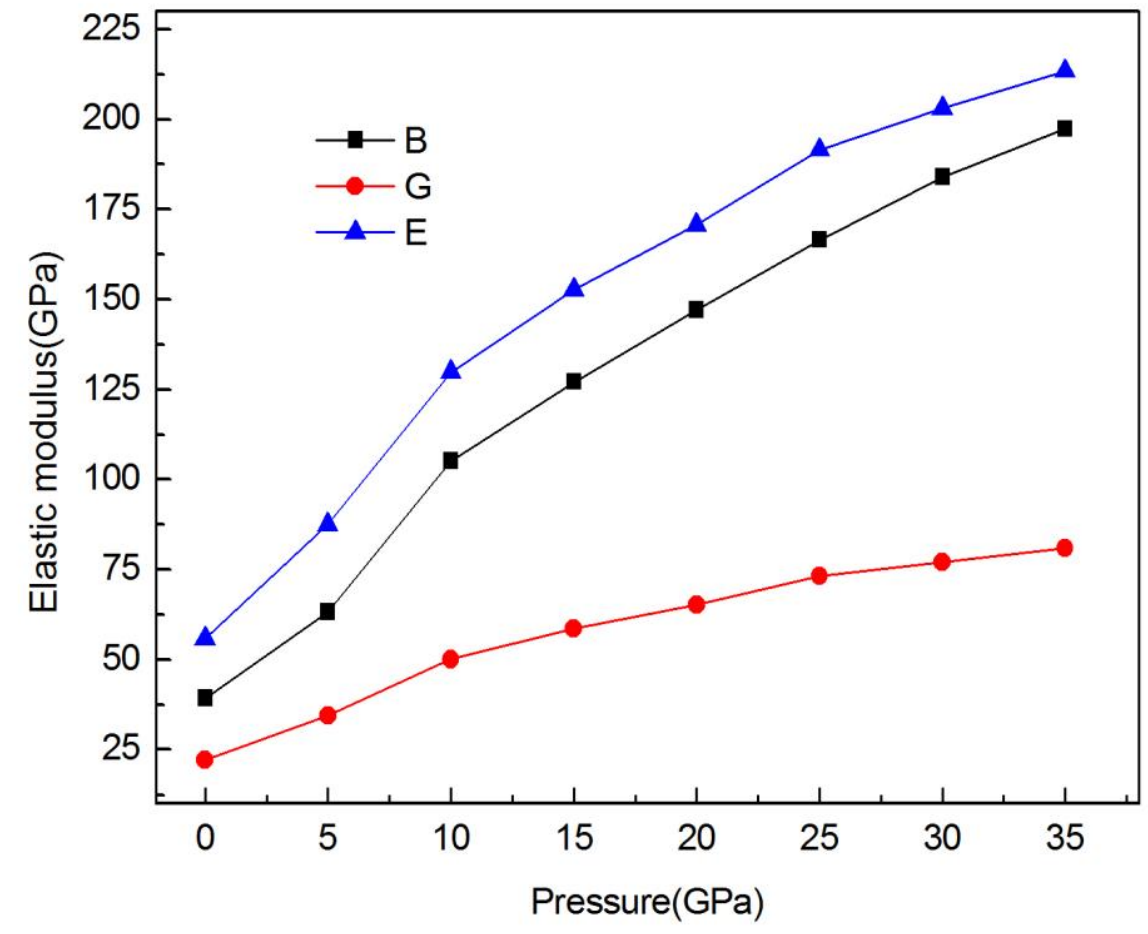

Fig. 4. The variations of the calculated bulk modulus, shear modulus and Young's modulus for the $\mathrm{Sn}_{2} \mathrm{~S}_{3}$ with pressure. 


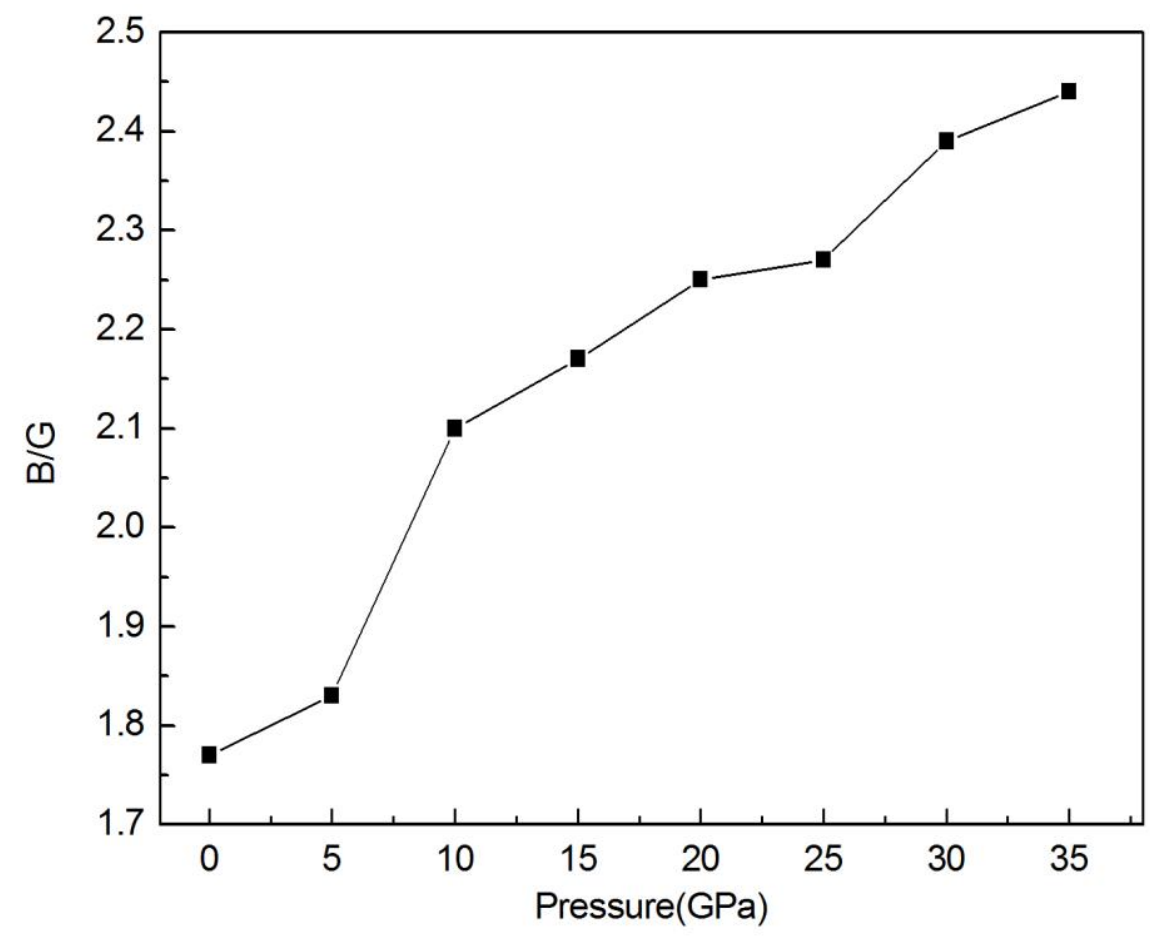

Fig. 5. The $B / G$ ratios of $\mathrm{Sn}_{2} \mathrm{~S}_{3}$ as a function of pressures.

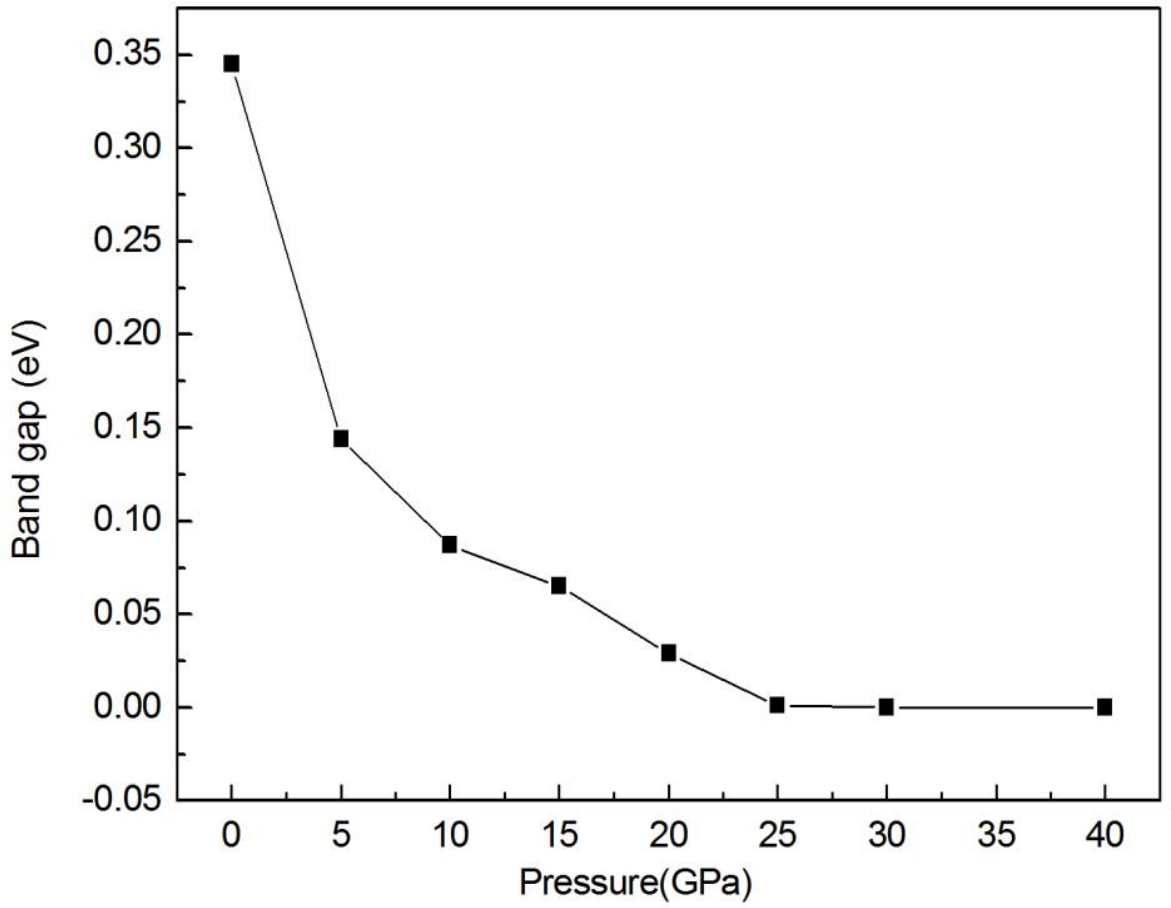


Fig. 6. The pressure dependence of the calculated band gap of $\mathrm{Sn}_{2} \mathrm{~S}_{3}$ after correction.

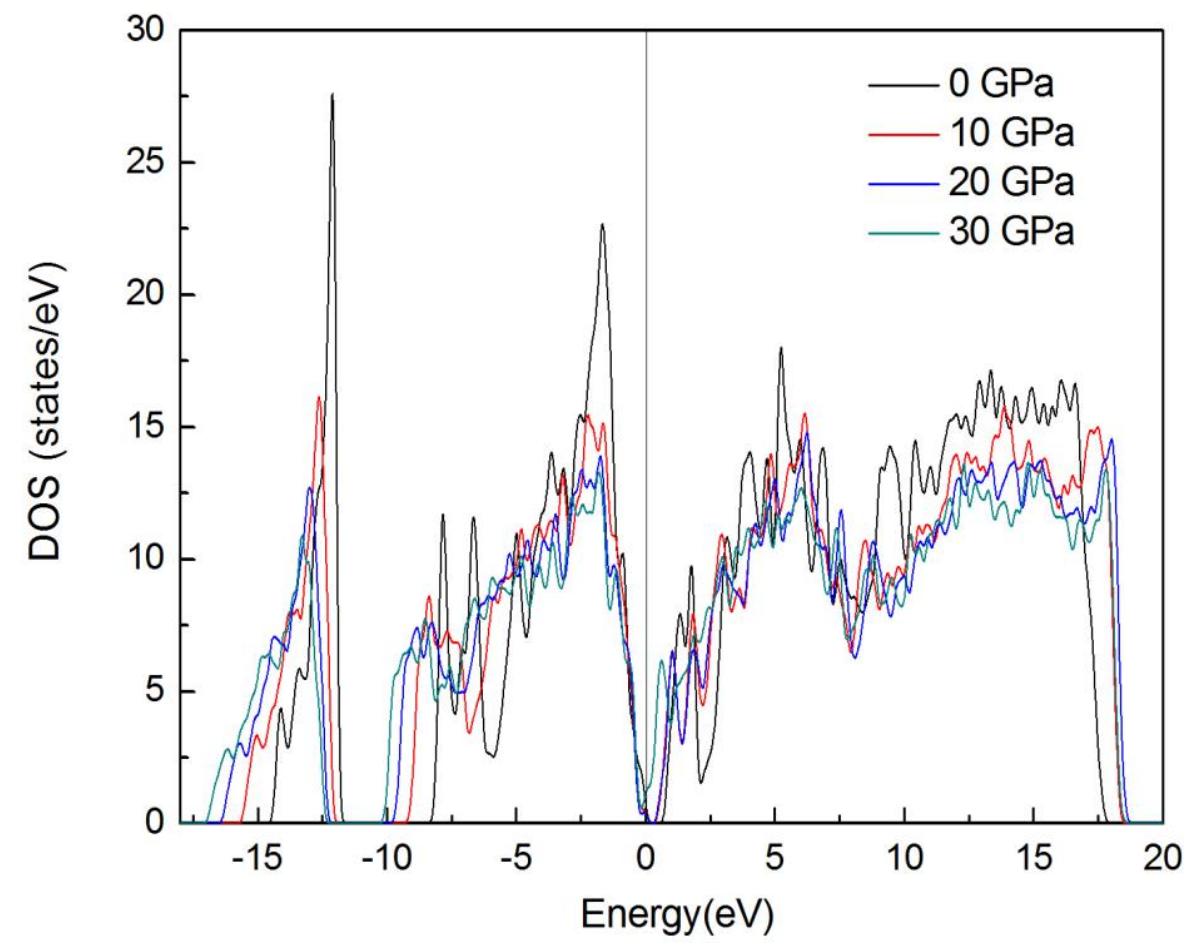

Fig. 7. The total density of states (DOS) of $\mathrm{Sn}_{2} \mathrm{~S}_{3}$ under pressure $(0,10,20$ and $30 \mathrm{GPa})$.
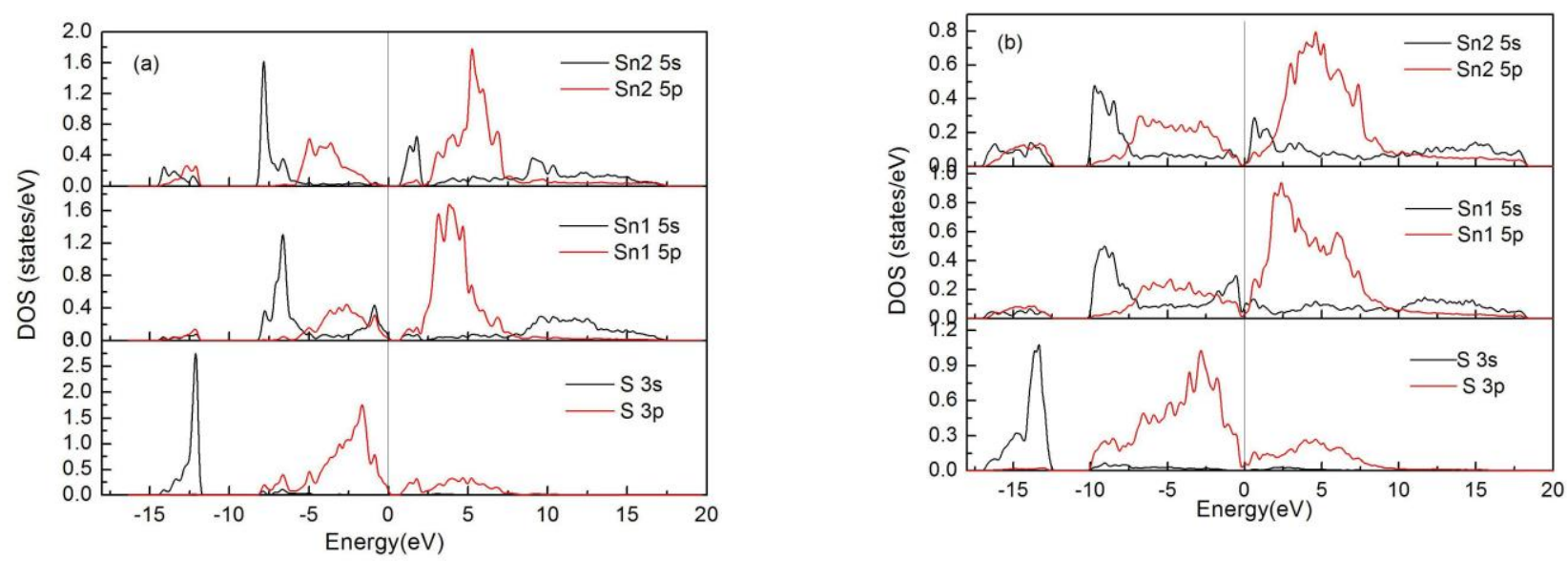

Fig. 8. The partial density of states (DOS) of $\mathrm{Sn}_{2} \mathrm{~S}_{3}$ under pressure ((a) $0 \mathrm{GPa}$ and (b) $30 \mathrm{GPa}$ ). 


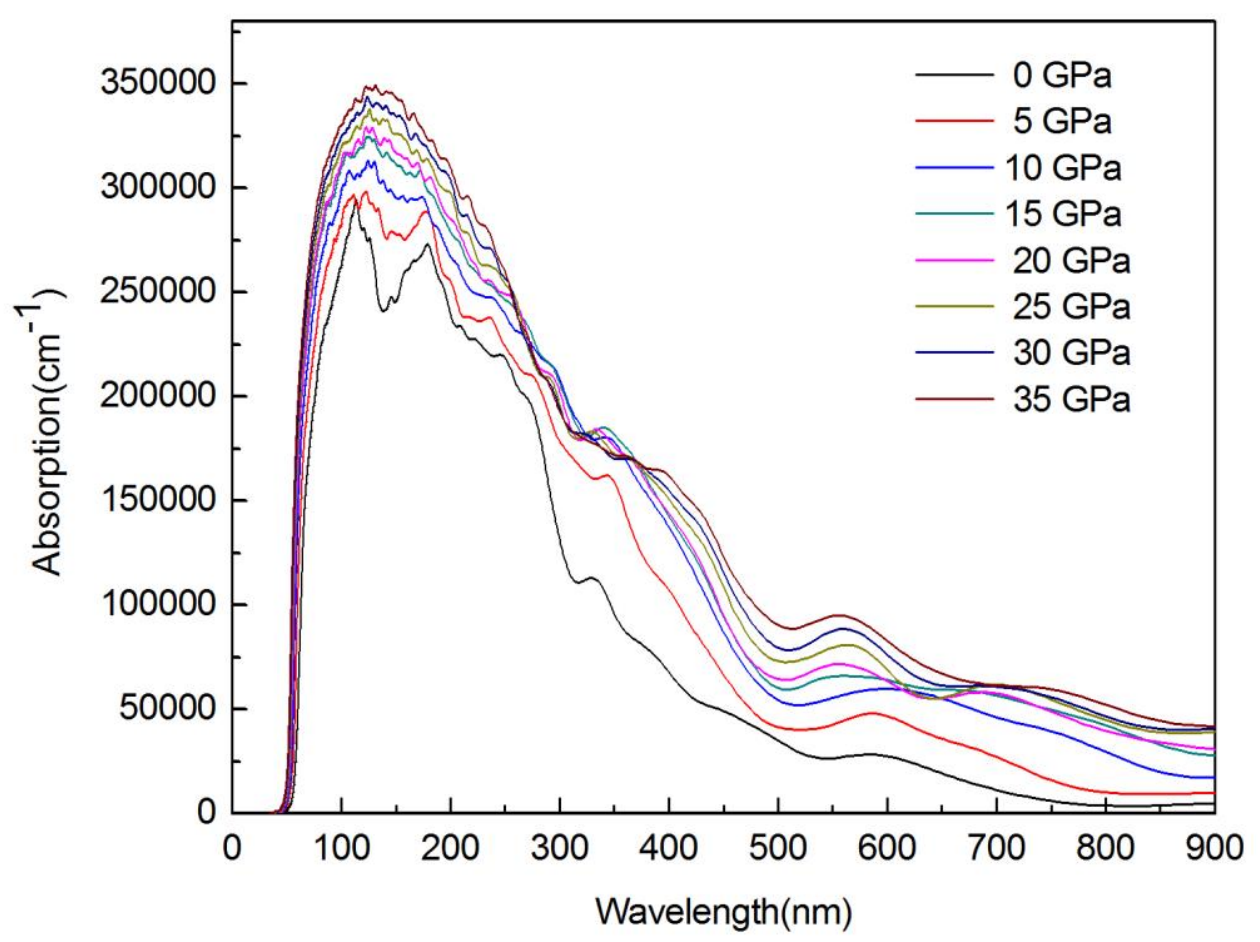

Fig. 9. The absorption spectra of $\mathrm{Sn}_{2} \mathrm{~S}_{3}$ as a function of wavelength at various pressures. 
Table 1 The calculated equilibrium lattice contants, equilibrium volume of $\mathrm{Sn}_{2} \mathrm{~S}_{3}$. The previous experimental and theoretical results are also listed.

\begin{tabular}{cccccc}
\hline & Approximation & $\mathrm{a}(\AA)$ & $\mathrm{b}(\AA)$ & $\mathrm{c}(\AA)$ & $V\left(\AA^{3}\right)$ \\
\hline This work & PBE & 9.134 & 3.820 & 14.254 & 497.39 \\
& PBEsol & 8.831 & 3.812 & 13.779 & 463.91 \\
Experimental[12] & - & 8.869 & 3.748 & 14.021 & - \\
Theoretical [28] & PBEsol & 8.80 & 3.77 & 13.83 & - \\
Theoretical [29] & HSE06 & 8.82 & 3.72 & 13.69 & - \\
\hline
\end{tabular}

